\title{
Phytochemicals in cancer prevention: modulating epigenetic alterations of DNA methylation
}

\author{
Sevinci Pop $\mathbb{~}$ - Ana Maria Enciu • Isabela Tarcomnicu • Elvira Gille • \\ Cristiana Tanase
}

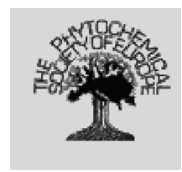

(2)

Received: 31 December 2018/ Accepted: 3 July 2019/Published online: 10 July 2019

(C) The Author(s) 2019

\begin{abstract}
Cancer can take many years to develop from initiation to progression. The long period of development might represent an opportunity to use multi-functional, multi-targeted preventive drugs to block or reverse tumorigenesis. One path to cancer prevention could be to target and reverse the early epigenetic alterations. Unlike genetic mutations, they are potentially reversible and can be restored to their normal state. Epidemiological studies have revealed the close link between rich diets in bioactive compounds and the low incidence of different types of
\end{abstract}

S. Pop $(\bowtie)$ - A. M. Enciu - C. Tanase

"Victor Babes" National Institute of Pathology, Splaiul Independentei 99-101, 050096 Bucharest 5, Romania e-mail: spop@ivb.ro

\section{A. M. Enciu}

Cell Biology and Histology Department, "Carol Davila, University of Medicine and Pharmacy “, Eroilor Sanitari 8, 050474 Bucharest 5, Romania

I. Tarcomnicu

SC Cromatec Plus SRL, Petre Ispirescu Street 1C, 077167 Tancabesti, Ilfov, Romania

E. Gille

National Institute of Research and Development for Biological Sciences/"Stejarul" Biological Research Centre, Alexandru cel Bun Street 6, 610004 Piatra Neamt, Romania

C. Tanase

Faculty of Medicine, "Titu Maiorescu" University, Calea

Vacaresti 187, 004051 Bucharest 4, Romania cancer. Thus, the study regarding the impact of bioactive nutrients on the epigenome has become widespread, with focus on the modulation of epigenetic mechanisms of gene expression, such as genomic DNA methylation. Following altered activity and expression of DNA methyl transferases and ten-eleven translocation enzymes, different types of cancers exert local DNA hypermethylation of gene promoters of tumor suppressor genes or of non-coding RNAs (microRNAs and long-noncoding RNAs), as well as global hypomethylation. Recently, the potential of phytochemicals to modulate epigenetic events in human health has become evident, although specific molecular mechanisms are still unclear. Phytochemicals and other bioactive dietary compounds can restore global and gene-specific promoter DNA methylation patterns by reactivating DNA methyltransferases or by providing the provision of methyl groups. Several natural products, such as EGCG, curcumin, sulforaphane, have shown DNMT inhibitory activity, but this property needs more in-depth investigations. This review focuses on the impact of modified DNA methylation pattern on early carcinogenesis and summarizes the effects/mechanism of phytochemical interventions on this type of epigenetic alterations.

Keywords Bioactive compounds . Chemoprevention · DNA hypermethylation . Epigenome $\cdot$ Sulforaphane 


\section{Abbreviations}

$5-\mathrm{hmC}$

5-Hydroxymethylcytosine

5-mC

APC

5-Methylcytosine

Bax

BRCA1

Adenomatous polyposis coli

CCND2

BCL2 associated X

CDKN2A

COMT

CYP

DACT2

DAPK

DKK1

DNMTs

EGCG

EZH2

GSTP1

H3K27

H4K16ac

HATs

HDAC

KDM4C

LINE

LKB1

MBD

$\mathrm{MeCP} 2$

MGMT

MLH1

MSH2

NQO1

Nrf2

PRC2

RASSF1

RB1

SAH

SAM

SNF

SWI/SNF

TDG

TET

TMS1

TP73

S-adenosylhomocysteine
Breast cancer 1

G1/S-specific cyclin-D2

Cyclin-dependent kinase inhibitor 2A

Catechol- $O$-methyltransferase

Cytochromes P450

Dishevelled binding antagonist of beta catenin 2

Death-associated protein kinase 1

Dickkopf-related protein 1

DNA methyltransferases

Epigallocatechin-3-gallate

Enhancer of zeste homolog 2

Glutathione S-transferase P1

Histone 3 lysine 27

H4 acetylated at lysine 16

Histone acetyltransferases

Histone deacetylase IDH2: isocitrate

dehydrogenase 2

Lysine-specific demethylase 4C

Long interspersed nuclear element

Liver kinase B1/serine/threonine kinase

11 (STK11)

Methyl-CpG-binding proteins

Methyl-CpG binding protein 2

O6-methylguanine-DNA-

methyltransferase

$$
\text { MutL alpha1 }
$$

MutS protein homolog 2

$\mathrm{NAD}(\mathrm{P}) \mathrm{H}$ : quinone oxidoreductase 1

Nuclear factor (erythroid-derived 2)-like 2

Polycomb repressive complex 2

Ras association domain-containing protein 1

\section{Retinoblastoma1}

S-Adenosyl-methionine

Sulforaphane

Switch/sucrose nonfermenting (chromatin remodeling complexes)

Thymine-DNA glycosylase

Ten-eleven translocation enzymes

Target of methylation-induced silencing Tumor protein $\mathrm{p} 73$

\section{Introduction}

Cancer is a deadly disease, affecting human health worldwide and causing a huge impact on economy and society. According to the latest World Health Organization (WHO) global report, cancer is responsible for an estimated 9.6 million deaths in 2018, and expected to increase with 20 million new cases by 2025. Around one-third of these deaths are caused by the five leading behavioral and dietary risks: high body mass index, low fruit and vegetable intake, lack of physical activity, tobacco use, and alcohol consumption (https://www.who.int).

The development of cancer is a complex, multifactorial process characterized mainly by genetic mutations and epigenetic alterations. Interestingly, only $5-10 \%$ of all cancers are caused by inherited genetic mutations, whereas most cancers are triggered by environmental and lifestyle factors that can induce epigenetic changes in normal cellular development and function (Anand et al. 2008).

Cancer is a disease that can take many years to develop, from initiation to progression. For example, all the common epithelial cancers (lung, colorectal, breast, prostate, pancreas and ovary) have a long latency period, often 20 years or more. By the time they are clinically detectable, the cells may harbor hundreds of mutations in different genes (Sporn 2011). The long-term development of certain types of cancer could represent a major opportunity to use multifunctional, multi-targeted preventive drugs in order to block or reverse cancer-related modified cells. One path for cancer prevention could be to target and reverse the early epigenetic alterations that, unlike genetic mutations, are potentially reversible and can be restored to their normal state.

The epigenetic mechanisms are regulating gene expressions through genomic DNA methylation, histone post translational modifications, chromatin remodeling, and expression of non-coding RNAs (microRNAs and long non-coding RNAs). Each epigenetic mechanism is controlled by specific protein classes which attach, remove, or maintain specific chemical groups that constitute epigenetic marks for activation or inactivation of the gene transcription. The link between epigenome (epigenetic regulatory proteins and chemical marks), epigenetic mechanisms and gene expression form a complicated "feedback" 
network that regulates and organizes cellular functioning at the molecular level (Dawson and Kouzarides 2012). When this regulatory circuit is discontinued by internal or external factors, normal physiological functions are affected, leading to tumor initiation process (Timp and Feinberg 2013).

Recent advances made in epigenetic field and cancer research showed that genetic and epigenetic mechanisms are not separate events in cancer; they interconnect and influence each other during tumorigenesis (You and Jones 2012). Alterations in epigenetic mechanisms can lead to genetic mutations; genetic mutations in epigenetic regulators lead to an altered epigenome (Timp and Feinberg 2013). Furthermore, evidence have suggested that epigenetic modifications might occur early in tumorigenesis and some of them even precede genetic mutations during cancer initiation (Feinberg et al. 2006). The abnormal proliferation of cells, due to accumulation of genetic and epigenetic aberrations, causes deregulation of major cellular processes, including cell cycling, DNA damage response, differentiation, and apoptosis.

Epidemiological studies (Zamora-Ros et al. 2014; Edmands et al. 2015; Sun et al. 2017) revealed that there is a close link between rich diets in bioactive compounds and the low incidence of different types of cancer. Over the recent years, studying the effects of bioactive nutrient treatment on the epigenome has become widespread, and it is currently certain that they can modulate epigenetic mechanisms of gene expression, such as genomic DNA methylation, acetylation or methylation of lysine residues from histones $\mathrm{H} 3$ and $\mathrm{H} 4$. Changes in DNA methylation have been recognized to be among the most common molecular alterations in human neoplasia and hypermethylation of gene-promoter regions is being revealed as one of the most frequent mechanisms of gene function loss.

This review focuses on the impact of modified DNA methylation pattern on early carcinogenesis and summarizes the effect/mechanisms of phytochemical interventions on this type of epigenetic alteration. The relationships between changes in DNA methylation pattern and lack of bioactive compounds intake, as well as the benefits of phytochemicals as prevention and/or early intervention in cancer, are also discussed.

\section{DNA methylation as epigenetic regulator of gene expression}

DNA methylation is the major epigenetic mechanism that provides a stable and reversible mechanism for gene silencing; it plays an important role in regulating gene expression, chromatin architecture and chromosome stability.

DNA methylation at the 5 position of cytosine (5$\mathrm{mC}$ ) is a key epigenetic mark that is critical for various biological and pathological processes. It consists in the addition of a methyl group from the universal methyl donor, S-Adenosyl-methionine (SAM), to the cytosine at the $\mathrm{CpG}$ dinucleotide residues. The members of the DNA methyltransferase (DNMT) family directly catalyze the addition of methyl groups onto DNA and are the major players as epigenetic modifiers. DNMT3A and DNMT3B are de novo methyltransferases by initiating DNA methylation and establishing the methylation patterns independently of replication. DNMT1 maintains the original pattern of DNA methylation in a cell lineage manner, and has the ability to repair DNA methylation (Mortusewicz et al. 2005). The maintenance mechanism mediated by DNMT1 is crucial to ensure the faithful reestablishment of 5-mC on the newly synthesized strand after DNA replication (Mortusewicz et al. 2005). Moreover, there is an active cooperation between all three enzymes in order to maintain DNA methylation at densely methylated regions, repetitive elements, and imprinted genes (Liang et al. 2002).

The $\mathrm{CpG}$ dinucleotides, known as $\mathrm{CpG}$ islands, are preferentially located in the proximal promoter end of approximately $60 \%$ of genes in the human genome. Unmethylated $\mathrm{CpG}$ islands correspond to either active transcription or a poised state, where genes can be expressed if the appropriate molecular signals are present (Suzuki and Bird 2008). In addition, large methylated domains are found predominantly in the long interspersed and tandem repetitive sequences that represent approximately $70-90 \%$ of the $\mathrm{CpG}$ dinucleotides in the entire genome (Rollins et al. 2006). These methylation patterns of the genome are vital for both chromosomal and genomic stability, possibly through the repression of retroviral transposons (Jones 2012). Consequently, the epigenetic mechanism through DNA methylation facilitates the organization of the genome into active (euchromatin) and inactive 
regions (heterochromatin) with respect to gene transcription.

DNA methylation is essential for normal mammalian development, function and differentiation by its epigenetic control of protein-coding RNAs or noncoding RNAs expression.

The 5-mC epigenetic mark is chemically stable and its presence at the promoter sites induces transcriptional inhibition by sterically blocking the further binding of transcription factors (Deaton and Bird 2011). Sequentially, the MBD proteins are recruited at methylated DNA and further facilitate the formation of compact, inactive heterochromatin. The unmethylated $\mathrm{CpG}$ sites bind switch/sucrose nonfermenting (SWI/SNF) chromatin remodeling complexes, histone acetyltransferases (HATs) and histone methyltransferases (HMTs), which label the chromatin with histone transcriptionally active marks (Bannister and Kouzarides 2011). The sequential recruitment of different protein complexes, followed by histone acetylation, demonstrated that the formation of open transcriptionally active chromatin is a dynamic process where layers of epigenetic regulators participate to the gene expression mechanism (Memedula and Belmont 2003; Bintu et al. 2016).

For decades, DNA methylation has been considered to be a non-reversible reaction, until the discovery of 5 hydroxymethylcytosine (5-hmC) and the TET family of enzymes.

The active demethylation consists of a series of successive oxidation reactions catalyzed by TETs. First, $5-\mathrm{mC}$ is converted to $5-\mathrm{hmC}$; afterwards, the methyl group is removed by a TDG-mediated base excision mechanism (Oswald et al. 2000; Weaver et al. 2004; Wu and Zhang 2017).

The 5-hmC epigenetic mark is also associated with differentiation and normal development, (Ficz et al. 2011) and the levels and distribution of 5-hmC might vary in different tissues, with the highest accumulation being found in the brain (Chen and Riggs 2011; Wu and Zhang 2017). While 5-mC is associated with closed heterochromatin, 5-hmC is associated with DNA demethylation and an open active chromatin state.

The role of $5-\mathrm{hmC}$ in gene expression regulation has still not been fully elucidated. The unique genomic distribution patterns of TET1 and 5-hmC at the transcription starting sites and promoters, as well as gene bodies, are suggesting that they might regulate gene expression through modulating chromatin accessibility or by inhibiting repressor binding (Williams et al. 2011; Wu and Zhang. 2017). As an epigenetic reader protein, methyl-CpG binding protein 2 (MeCP2) has similar affinity to both DNA epigenetic marks (Mellén et al. 2012), but it has been observed that the 5 -hmC level is negatively correlated with $\mathrm{MeCP} 2$ abundance. One explanation could be that the binding of $\mathrm{MeCP} 2$ to 5-mC can possibly hinder the production of 5-hmC (Mellén et al. 2012). Therefore, 5-hmC and $\mathrm{MeCP} 2$ might constitute a cell-specific epigenetic mechanism for the regulation of gene expression and remodeling the chromatin structure. The interconnection between DNA methylation and the demethylation processes is exemplified in Fig. 1, where the enzymatic processes catalyzed by the epigenetic regulator proteins (DNMTs, TETs) are changing other epigenome marks $(5-\mathrm{mC}, 5-\mathrm{hmC})$.

DNA methylation is a reversible, enzymatically controlled mechanism of gene expression, involved, among other processes, in normal embryogenesis, tissue differentiation and chromosome stability. Modifications in any of the layers controlling this epigenetic process can lead to carcinogenesis, as discussed in the following section.

\section{Epigenetic alterations in carcinogenesis: DNA methylation}

Cancer methylation has been characterized by global hypomethylation together with local, de novo promoter $\mathrm{CpG}$ islands hypermethylation (Jones and Baylin 2007; Sharma et al. 2010), including those of classic tumor suppressors (Shen and Laird 2013). Furthermore, some local variations in methylation pattern at only several key genome loci are sufficient for cancer initiation (Plass et al. 2013). Importantly, the altered patterns of DNA epigenetic marks (5-mC, 5 -hmC) are frequently accompanied by a critical imbalance in transcriptional programs involving differentiation and stem cell maintenance, thereby could participate to tumor initiation and sustaining cancer cells growth (Jones and Baylin 2007).

Actually, a series of studies showed that the DNA methylation is a driver of tumorigenesis and that cancer cells suffer additional epigenetic alterations which are essential for cancer cell survival (Baylin and Herman 2000; De Carvalho et al. 2012). Furthermore, 


\section{DNA methylation/demethylation process}

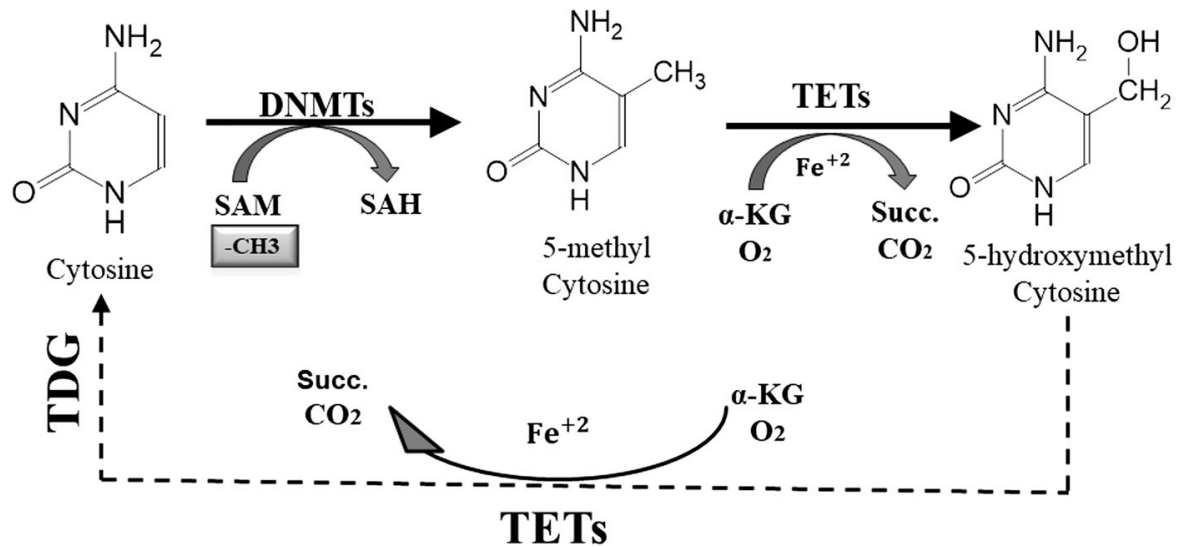

Fig. 1 DNA methylation and demethylation process. DNA methylation occurs at the $5^{\prime}$ position of cytosine, within $\mathrm{CpG}$ dinucleotides. DNMTs catalyze the transfer of the methyl group to cytosine and generate 5-mC using SAM as methyl donor and producing SAH. DNA demethylation is a multi-step oxidation process catalyzed by TETs methylcytosine dioxygenases family

the fact that DNA hypermethylation could be an early event in carcinogenesis is supported by the finding that adjacent normal tissues also harbor altered DNA methylation patterns (Taby and Issa 2010). Only

$15 \%$ of the genes methylated in cancer samples were actively transcribed in normal tissue; moreover, they were already inactivated by methylation in precancerous tissue (Keshet et al. 2006).

\section{Local DNA hypermethylation}

The list of aberrantly methylated genes in cancer is steadily growing, including those with major impact on cellular pathways involved in carcinogenesis: detoxification (GSTP1), DNA repair (MGMT, BRCA1, MLH1), cell cycle (CDKN2A/p16-INK4, CCND2, RB1), Ras signaling (RASSF1), Wnt signaling (APC, DKK1), apoptosis (DAPK, TMS1, TP73) and so on. Most of these genes are found to be inactivated in many types of cancer and the DNA methylation of promoter's gene could be considered as early event in tumor initiation, which could represent the major target for cancer prevention.

For example, the hypermethylation and silencing of the intracellular detoxification enzyme GSTP1 is that uses $\mathrm{Fe}^{2+}$ and $\alpha$-ketoglutarate as cofactors or substrates, and generates succinate and $\mathrm{CO}_{2}$. In the first step of demethylation process, the $5-\mathrm{mC}$ is converted to 5 -hmC, and after several oxidation reactions the methyl group can be remove by TDG-mediated base excision repair mechanism

considered a molecular hallmark of prostate cancer and was already implemented in clinical diagnosis. The silenced GSTP1 was found at the earliest stages of prostate cancer initiation and was observed in more than $\sim 90 \%$ of the tumors, and in several other types of cancer (Kim et al. 2011; Witte et al. 2014). The loss of GSTP1 enzymatic detoxification activity may explain the well-known sensitivity of human prostatic carcinogenesis to environmental factors and the demethylation of GSTP1 promoter could become a target for epigenetic chemoprevention (Jerónimo et al. 2011).

Similarly, methylation mediated silencing was reported in other important DNA repair genes, such as MGMT, BRCA1, BRCA2 and MSH2 at a pancancer level, and are frequently observed in multiple cancer types, for instance lung, gastric, colorectal, leukemia, brain, liver, breast, and prostate (Witte et al. 2014). The inactivation of genes in DNA repair pathways will further propagate the carcinogenic state by allowing cells to accumulate additional genetic lesions. MGMT, which normally protects from mutations occurring at guanine bases, is silenced by hypermethylation events and often occurs early in tumorigenesis (Witte et al. 2014). The inactivation of 
MGMT is predominantly epigenetic, for example its promoter is methylated in $44 \%$ of human esophageal squamous cell carcinomas, while appearing mutated in only $17.5 \%$ of the patients (Keshet et al. 2006; Weisenberger 2014). These results suggest that a primary epigenetic defect in mismatch repair mechanisms can accelerate the rate of accumulation for additional mutations in cancer cells.

In addition, the silencing of transcription factors could indirectly silence or downregulate a large number of other genes. For example, silencing a key tumor suppressor p16 (encoded by the CDKN2A gene in humans) is related with aberrant promoter hypermethylation and it is a common epigenetic mark in human cancers. Mice bearing a hypermethylated p16 promoter had a higher incidence of developing spontaneous cancer during ageing. But when the mice carried inactivating germline mutations in one allele of p16 and epigenetic alterations in the other allele, they showed early onset of tumors and shorter survival time (Yu et al. 2014). Put together, these data demonstrate that epigenetic mutations are able to act as driver events in tumor initiation and progression.

Interestingly, dysregulation of DNA methylation status at promoters of non-coding RNAs (miRNAs and lncRNAs) could also promote carcinogenesis (Kozaki and Inazawa 2012; Pop et al. 2018). Aberrant microRNA expression in cancer has been associated with epigenetic regulation, such as DNA methylation and histone modifications. It is estimated that the transcription of $10 \%$ of all microRNA species is controlled by DNA methylation (Kozaki and Inazawa 2012) and approximately 50-70\% of microRNA genes are located at fragile genomic sites that are frequently affected during carcinogenesis (Starczynowski et al. 2011). Also, microRNAs control and regulate expression of major epigenetic modifier proteins involved in DNA methylation processes, including DNMTs and TETs. Several studies have demonstrated that alterations in the expression of miRNAs are prominent events during the early stages of liver carcinogenesis and may predict susceptibility to cancer development (Anwar and Lehmann 2014). In vivo studies showed that a methyl-deficient diet induced hepatocellular carcinogenesis associated with global DNA hypomethylation, and with changes in several miRNA expression, which could be reversed by restoring dietary methyl donors (Parasramka et al. 2012).
Global DNA hypomethylation

The global lower methylation level (hypomethylation) is a common epigenetic alteration in cancer, especially at Long Interspersed Nuclear Element (LINEs) regions, which are a group of retrotransposons widespread in human genome. They are translated into proteins that act as reverse transcriptase able to reproduce DNA copies, which are then relocated into new genomic sites. In human somatic cells, the LINE1 is heavily methylated and thus is mostly suppressed, maintaining the genomic stability by avoiding retrotransposition to other genomic loci (Lee et al. 2012). Several studies showed that LINE-1 is gradually hypomethylated during cancer progression, with the first signs of global methylation changes initiated early in carcinogenesis (Slotkin and Martienssen 2007; Kitkumthorn and Mutirangura 2011). Hypomethylation of LINE-1 triggers the active process of genome reorganization, and the relocations of these interspersed repetitive sequences are a source of endogenous mutagenesis and polymorphism in the premalignant and malignant cells (Kitkumthorn and Mutirangura 2011; Lee et al. 2012).

Also, the loss of 5-hmC is an epigenetic hallmark of aggressive tumors, such as melanoma, glioblastoma or ovarian cancer, with both diagnostic and prognostic implications (Tucker et al. 2018). The overexpression of active proteins IDH2 or TET2 in animal models for human melanoma was an efficient way to increase the 5-hmC level (Lian et al. 2012). Likewise, pretreatment with DNMTs inhibitors restored the 5-hmC patterns via enhanced levels of TET family enzymes, both in vitro and in vivo experiments (Tucker et al. 2018).

\section{Other epigenetic alterations}

There are approximately 40 epigenetic regulators that exhibit some form of alteration in cancer (Jones and Baylin 2007). The most prominent are somatic mutations in the proteins involved in DNA methylation and demethylation mechanisms. For example, somatic heterozygous mutations in DNMT3A are found in $\sim 20 \%$ of patients with acute myeloid leukemia (AML) and recent evidence suggest that at least some of these mutations exhibit dominantnegative effects by inhibiting the function of the wild-type DNMT3A allele (Kim et al. 2013). The 
epigenetic regulators expression could be directly altered in various cancers, but other mutated genes may impinge on the proper function of these enzymes. For example, recurrent mutations in the IDH1 and IDH2 alter their enzymatic activity and consequently the 2-hydroxyglutarate is produced, which may inhibit several dioxygenases, including TET2 and KDM4C (Feinberg et al. 2016). This indicates that not only the epigenetic factors are directly altered in tumorigenesis, but also that mutations in other pathways can have an impact on the regulation of gene expression. Genetic alterations in TET1 and TET2 have also been identified in leukemia and solid tumors ( $\mathrm{Wu}$ and Zhang 2017), with direct effects on epigenetic degradation of 5-hmC and 5-mC patterns. Coordinated epigenome changes can also be achieved via the interaction of multiple epigenetic regulators, which guide different enzymatic activities to the same locus. Recent results have demonstrated that the PRC2 complexes are recruited to specific DNA regions based on DNA sequence and transcription factor occupancy. The protein EZH2, that mediates repressive chromatin formation through the deposition of H3K27 methylation, and DNMT enzymes, act at the same locus for the coordination of repressive histone and DNA methylation marks (Viré et al. 2006). Thus, the crosstalk between the different layers of the epigenetic mechanism could amplify early epigenetic changes, leading to the development of cancer.

The overexpression of DNMTs represents a common feature in a variety of tumors, and results in local DNA hypermethylation and oncogenic activation. Highly expressed DNMT3A and DNMT3B have been found in a large number of patient specimens, with increased DNMT3A expression in hepatocellular carcinogenesis, where intense hypomethylated genomic regions were also observed (Zhao et al. 2010). Moreover, high expression levels of DNMT3B have been correlated with the epigenetic inactivation of BRCA1 in sporadic breast tumors (Butcher and Rodenhiser 2007). As a result, the overexpression of DNMTs in many cancers promoted local and global DNA methylation aberrations related with genomic instability and oncogenic pathways activation.

In conclusion, there are several epigenetic mechanisms related to DNA methylation patterns, from local hypermethylation of specific gene promoters to global DNA hypomethylation, with impact on cellular processes which dysregulated can lead to carcinogenesis, as we exemplified in Fig. 2.

Whether dysregulation of DNMTs and TETs enzymes can be modulated by phytochemicals in an efficient manner, in order to counteract various mechanisms acting in tumor initiation and progression, will be the topic of the following section.

\section{Phytochemicals in cancer prevention}

Cancer chemoprevention implies the use of dietary or pharmacological compounds to prevent, inhibit, or even reverse the process of carcinogenesis before clinical manifestation of the disease. Therefore, effective chemoprevention requires the use of compounds that inhibit specific molecular steps in the carcinogenic pathway, including the epigenetic alterations that are early and potentially reversible events. Substantial experimental evidence and epidemiological studies indicate the potential importance of dietary phytochemicals and nutritional factors in cancer prevention. Diets rich in fruits and vegetables could prevent at least $20 \%$ of all cancers (www.who.int). In addition, a recent study showed that more than $49 \%$ of all 175 small molecules approved for cancer therapy were natural products or directly derived from them (Newman and Cragg 2016). Natural products with bioactive components have gained increasing attention in cancer prevention and therapy, due to their compatibility with biological target sites and less induced toxicity to normal cells (Remely et al. 2015). Several preclinical studies have reported that many phytochemicals with anti-inflammatory, anti-oxidation and anti-proliferative properties can prevent cancer initiation and development by inducing apoptosis and activating antioxidant enzymes (Venkatachalam et al. 2016; Leone et al. 2017). However, the translation of chemopreventive properties of phytochemicals to clinical practice has not been yet achieved.

Recently, the potential of phytochemicals to modulate epigenetic events in human health has become evident, although specific molecular mechanisms are still unclear. Among the impressive number of phytochemicals with anti-tumoral properties, some polyphenols and organosulfur compounds are part of the dynamic interaction between the genome and the environment with specificity at physiological 


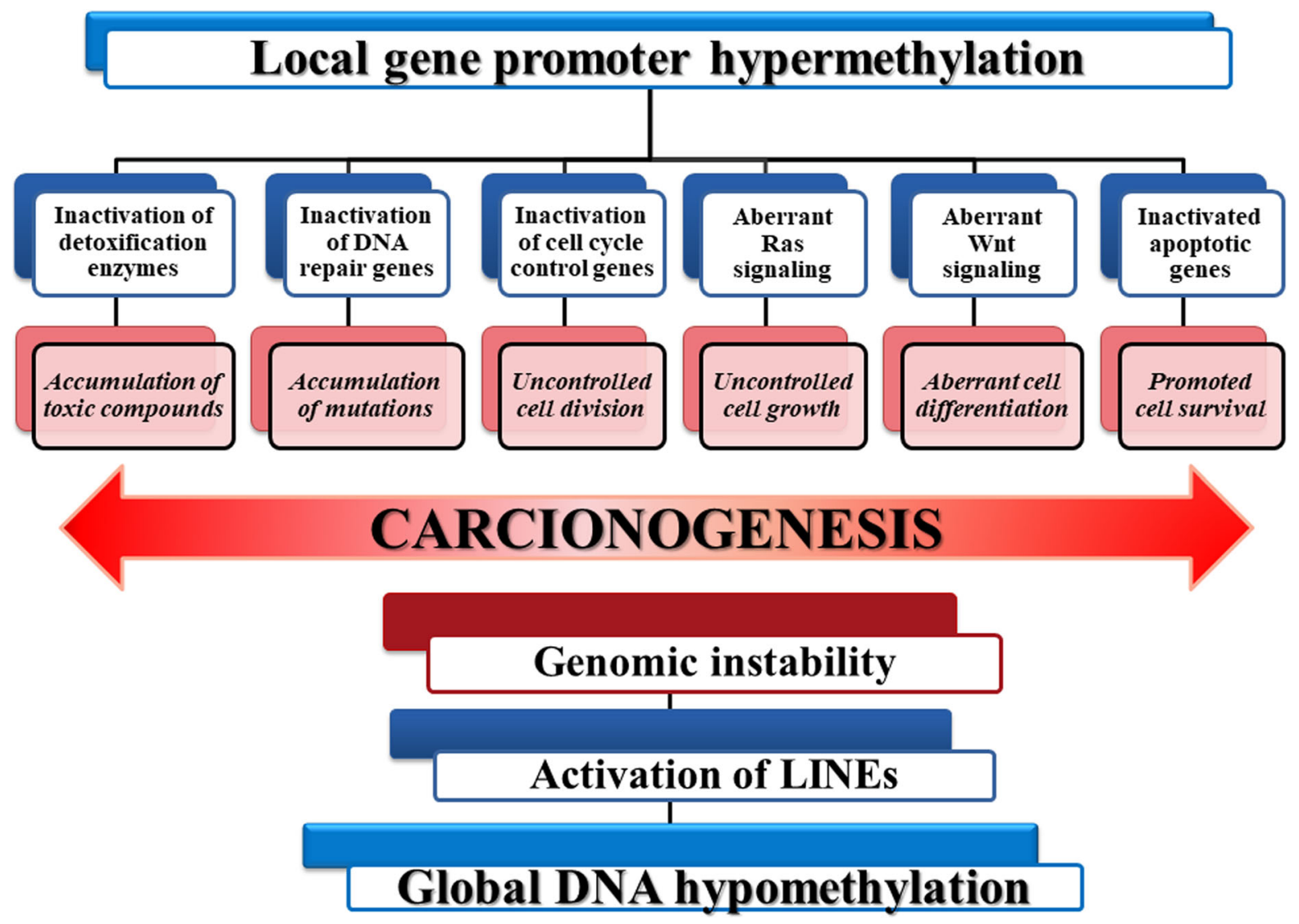

Fig. 2 Epigenetic alterations of DNA methylation in carcinogenesis. There are two main epigenetic changes of DNA methylation related to cancer initiation and developmentglobal DNA hypomethylation and local gene promoters hypermethylation. Each leads to specific events, such as

concentrations, and well known to modulate mechanisms underlying in human health.

DNA methylation dysregulations induced by poor nutrition

Recent studies have highlighted the cross-talk between cancer metabolism and the epigenome. Metabolites such as SAM, acetyl-coA, and AMP are required for epigenetic mechanisms such as DNA and histones methylation, histone acetylation or phosphorylation (Donohoe and Bultman 2012; Newman and Maddocks 2017). The metabolic pathways and enzymes that supply these key compounds are therefore critical for the maintenance and adaptation of the epigenome. Indeed, a diet with deficit in methionine decreases SAM levels, leading to diminished DNA activation of LINEs or, inactivation of genes involved in specific cellular processes: detoxification, DNA repair genes, and tumor suppressor gene respectively. Isolated or in summation, these alterations can eventually lead to cancer

and histone methylation with significant effects upon gene expression (Donohoe and Bultman 2012; Parasramka et al. 2012). The metabolism of folate, betaine, choline, and methionine are interrelated, and the deficiency of one nutrient can cause metabolic and functional disturbances. A diet poor in methyl donor contributors can have a rapid effect on global DNA methylation pattern. For example, within 1 week, the global DNA hypomethylation and increased levels of mRNA for oncogenes c-fos and c-myc were observed in liver tissues of Fischer rats fed with methionine and choline-deficient diet. After restoration of proper methyl donors rich diet, the global and local DNA methylation pattern returned to normal within 1-2 weeks (Niculescu and Zeisel 2002). In the case of longer exposure to methyl deficient nutrition (18-36 weeks), the epigenetic alterations of DNA 
methylation could not be reversed by reintroducing the animal models to the right diet. The global DNA hypomethylation pattern and altered hepatic foci in their liver were irreversible (Pogribny et al. 2006). These data provide further experimental evidence to demonstrate that epigenetic alterations may contribute to the initiation and promotion of liver carcinogenesis.

During methionine starvation, the other metabolic pathways, such as serine cycle could provide cofactors to recycle homocysteine to methionine. Indeed, the serine-dependent de novo ATP synthesis might support the conversion of methionine to SAM. In this case the ATP pool is reduced and this can have a direct impact on the rate of SAM generation and methylation of DNA (Maddocks et al. 2016). Recently, the specific contribution of one-carbon metabolism-dependent DNA methylation in pancreatic cancer has been explored. The loss of the serine-threonine kinase, LKB1, promotes tumorigenesis in KRAS mutant pancreatic cancer, accompanied by increased levels of global DNA methylation and increased expression of DNA methyltransferases for which SAM is a critical cofactor. This serine-dependent DNA methylation upon the loss of LKB1 in KRAS-mutant cells contributes to tumor growth, presumably through the activation of several oncogenes (Kottakis et al. 2016). Dietary and genetic perturbation of metabolic pathways could lead to dysfunctional DNA synthesis and DNA methylation, connecting directly the cancer metabolism to the epigenetic mechanism.

The cellular DNA methylation processes involve a series of catalytic reactions, which result in the generation of the principal methyl donor, SAM, followed by methyl group transfer reactions. As a consequence of methyl group transfer, SAM is converted to SAH, which binds to methyltransferases and induces product inhibition (Mortusewicz et al. 2005). Therefore, maintaining the proper ratio of SAM to $\mathrm{SAH}$ is a determinant factor for DNA methylation mechanism, since this ratio dictates methyltransferase activity in vivo. Disturbance of this system may be caused by dietary imbalances and in consequence the major epigenetic regulatory enzymes are affected, dysregulating DNA methylation pattern (Stefanska et al. 2012). In a pre-malignancy pathological condition, the appropriate consumption of a diet rich in methyl donor nutrients may interfere with early carcinogenesis events leading to cancer prevention.
The dietary phytochemicals may exert their chemoprevention activities by indirectly modulating DNMTs activities through altering the SAM/SAH ratio and having effects upon interference with cellular metabolism. The flavanol-rich diets contain polyphenols with catechol structures that can be methylated by catechol- $O$-methyltransferase (COMT) enzyme using SAM as a methyl donor (Bistulfi et al. 2010; Chen et al. 2010). This methylation reaction results in the demethylation of SAM and formation of SAH, which is a potent and selective inhibitor of DNA methyltransferase (Zhu et al. 2010). Phytochemicals and other bioactive dietary compounds can restore global and gene-specific promoter DNA methylation patterns by reactivating DNA methyltransferases or providing the provision of methyl groups. Therefore, phytochemicals could epigenetically modulate gene expression by changing the chromosomal integrity and stability with benefits on health conditions.

Phytochemicals modulating the epigenetic alterations of DNA methylation pattern

Numerous studies have demonstrated that certain dietary phytochemicals inhibit tumor growth by affecting epigenetic signaling pathways both in vitro and in vivo (Remely et al. 2015).

The dietary phytochemicals with epigenetic modulation activities of DNA methylation levels can be categorized in three group based on their mechanism of action (Ho et al. 2011): (1) the phytochemicals which directly donate the methyl group and act as cosubstrates in DNA methylation process; (2) the phytochemicals that indirectly modulate the DNMTs activity by affecting the methyl pool; and (3) the phytochemicals that act as direct DNMT enzyme inhibitors, which are amongst the most promising bioactive natural products candidates for cancer prevention and therapy.

Polyphenols are the largest class of plant secondary metabolites that are mainly found in fruits, vegetables, cereals, and beverages. Besides common antioxidant or anti-inflammatory activity, many polyphenols might modulate early epigenetic alterations related to cancer prevention. The polyphenol groups include phenolic acids (hydroxybenzoic and hydroxycinnamic acids), lignans, stilbenes, and flavonoids (Hardman 2014). 
Flavonoids are the most representative group of dietary polyphenols with diverse biological activities including anti-bacterial, anti-viral, analgesic, hepatoprotective, apoptotic, and estrogenic functions (Kumar and Pandey 2013). The chemoprevention activity of flavonoids might be mediated by certain epigenetic mechanisms, including modulation of DNA methylation status and histone methylation and acetylation (Jiang et al. 2015; Guo et al. 2018; Khan et al. 2018). The flavone apigenin can restore the silenced status of Nrf2 gene in skin epidermal cells by reducing the expression of three DNA methyl transferase proteins (DNMT1, DNMT3A, and DNMT3B) as well as the expression of some HDACs (Shukla and Gupta 2010). In addition, apigenin, together with another flavone, luteolin, has been reported to act synergistically to modulate the DNMT activity in esophageal squamous cell carcinoma line (Busch et al. 2015). Research suggests that a diet rich in flavones might decrease the risk of certain cancers, including breast, digestive tract, skin, and prostate cancer ( $\mathrm{Li}$ and Tollefsbol 2010; Shukla and Gupta 2010).

Some flavonoids have a selective DNMTs inhibitory activity, for example kaempferol inhibits DNMT1 and DNMT3B but not DNMT3A' enzymatic activity in bladder and CRC cancer (Banerji 2017; Lu et al. 2018).

Genistein is the most potent DNMT inhibitor amongst isoflavones, capable to reactivate methylation silenced genes such as RARb, p16INK4a, and MGMT in esophageal squamous carcinoma and prostate cancer cells (Fang et al. 2005; Dietz et al. 2016). Besides, genistein treatment of benign and tumor breast cells depletes human telomerase reverse transcriptase (hTERT) activity, the catalytic subunit of telomerase, which is overexpressed in $90 \%$ of cancers, through epigenetic modulation that involves decreasing the DNMTs expression levels and concomitant with hyper-methylation of $\mathrm{H} 3 \mathrm{~K} 9 \mathrm{me} 3$ and hypomethylation of $\mathrm{H} 3 \mathrm{~K} 4 \mathrm{me} 2$ chromatin marks ( $\mathrm{Li}$ et al. 2009).

The major catechin from green tea, EGCG, exerts its chemoprevention effect by blocking cell proliferation and transformation and promoting apoptosis and cell cycle arrest in several human cancer cell lines including leukemia, melanoma, breast cancer, lung, and colon (Singh et al. 2011; Schramm 2013). The molecular mechanism underlying EGCG chemoprevention action is related with the regulation of several signal transduction pathways including: MAPK, PI3K/AKT, Wnt, Notch, and NF- $\kappa \mathrm{B}$ (Pandey et al. 2010; Moseley et al. 2013; Schramm 2013; Khan et al. 2018). Additionally, EGCG has been demonstrated to induce the increase of tumor suppressor expression, such as: p53, p21, p16 and Rb with certain roles in chemoprevention (Pandey et al. 2010; Du et al. 2012). Moreover, EGCG possesses a chemopreventive effect against a broad spectrum of carcinogens by inhibiting the chemical induced colon, liver and skin carcinogenesis in several animal models (Henning et al. 2013).

EGCG exerts its epigenetic modulator capacity of DNA methylation processes indirectly, by acting as a substrate for COMT catalyzed methylation reaction (Bistulfi et al. 2010) or directly by inhibiting DNMT1 and DNMT3A enzymatic activities through blocking their catalytic sites. Molecular docking studies indicate that the gallic acid moiety of EGCG can accommodate in the hydrophilic active pocket of DNMT1 (Lee et al. 2005). Fang et al. demonstrated that EGCG binds to DNMT and competitively inhibits the enzymatic activity yielding to the reactivation of methylation-silenced genes in prostate cancer cells (Fang et al. 2003). In addition, treatments of different PCa cell lines with EGCG have determined a doseand time-dependent re-expression of GSTP1 enzyme concomitantly with the down-regulation of DNMT1 (Naponelli et al. 2017). Recent studies have demonstrated that EGCG induces epigenetic changes modulating hTERT activity through inhibition of DNMT and HAT activities (Li and Tollefsbol 2010; Du et al. 2012). All these data support the idea of EGCG as a key active nutrient for cancer inhibition through epigenetic control; however polyphenolic catechins generally exhibit poor oral bioavailability. Further investigation is required to improve EGCG absorption and metabolic biotransformation, in order to increase its potential effect in cancer prevention and therapy through epigenetic modulation.

Curcumin (diferuloylmethane) is a polyphenolic compound derived from turmeric (Curcuma longa Linn) with remarkable medicinal properties, mainly with anti-inflammatory and anti-cancer effects. Curcumin has been shown to modulate multiple intracellular pathways associated with proliferation, survival, invasion, apoptosis, and inflammation (Park et al. 2013; Jiang et al. 2015, Guo et al. 2018). In silico molecular docking studies revealed that curcumin can 
block or inhibit the catalytic site of DNMT1, thus resulting in decreased enzymatic activity. In vitro experimental studies validated the DNMT1 and DNMT3B inhibition activity of curcumin in several human cancer cell lines (Jiang et al. 2015; Guo et al. 2018). Similarly, in vitro and in vivo experiments showed that curcumin and its synthetic analogue (FN1) were able to restore the activity of Nrf2 gene by hypomethylation of its promoter and through inhibition of DNMTs activity, hence activating anti-oxidant pathways (Li et al. 2016a, b).

Several examples of phytochemicals involved in epigenetic modulation of DNA methylation dysregulation and chemoprevention in different carcinogenesis are presented in Table 1, such as well-known resveratrol, quercetin and others.

We will exemplify next with one phytochemical which exerts its epigenetic modulator capacity on various types of epigenetic alterations in carcinogenesis and could interfere with different layers of epigenetic mechanism, sulforaphane (SNF).

Sulforaphane belongs to Brassicaceae family and represent the most effective chemopreventive agent among isothiocyanate (ITC) group of organosulfur compounds. Many studies have shown that SFN is an effective chemopreventive agent that has anti-proliferative, anti-inflammatory, anti-angiogenic, and antioxidative effects, as well as induction of differentiation, apoptosis, and cell cycle arrest in several types of cancers (Cao et al. 2018). SFN induces its chemopreventive effects partly by activation of phase I CYP enzymes and phase II detoxification enzymes, leading to restored mitochondrial function and reduced lipid peroxidation (Kwon et al. 2007). In human breast, colon and hepatocellular carcinoma, the chemopreventive activities of SFN are mediated, at least in part, through Nrf2 pathway activation, which modulates phase 2 detoxification enzymes, including $\mathrm{NAD}(\mathrm{P}) \mathrm{H}$ : quinone oxidoreductase 1 (NQO1) and GST (Cao et al. 2018). A recent study demonstrates that SFN can activate the Nrf2 pathway in breast cancer cells, acting as an epigenetic modifier to regulate COMT expression to influence estrogen metabolism (Cao et al. 2018).

Numerous in vivo studies on murine models of colon, prostate, oral and pancreatic cancer showed the chemopreventive role of SNF by inhibiting tumor growth (Hsu et al. 2011). Interestingly, topical application of SFN for a long period of time inhibited chemical induced skin carcinogenesis in C57BL/6 mice, whilst no such chemopreventive effects of SNF were elicited in the Nrf2-deficient mice (Kwon et al. 2007).

There has been an increased interest in SFN recently, due to its potency to influence epigenetic processes through targeting key epigenetic modulators such as DNA methyltransferases and HDACs, which may lead to local or global alterations of epigenetic hallmarks resulting in subsequent gene transcription and expression level changes (Khan et al. 2018). Also, SFN modulates DNA demethylation by downregulation of the expression of DNMT1 and DNMT3B, subsequently leading to induced demethylation of cyclin D2 gene promoter and expression in cancer cells (Hsu et al. 2011). Similarly, in prostate cancer cells, SNF has been reported to be able to restore the expression of silenced GSTP1 by a mechanism involving promoter demethylation and increased histone acetylation. These effects are associated with increased expression of the CDKNs p21 and p27, which are negative cell cycle regulators (Hsu et al. 2011). Moreover, the SNF inhibition of the growth of prostate cancer PC-3 tumor xenografts could be correlated with inhibited HDAC activity. In human subjects, a single dose of $68 \mathrm{~g}$ broccoli sprouts decreased HDACs activity significantly in peripheral blood mononuclear cells (PBMC) at 3 and $6 \mathrm{~h}$ following consumption (Myzak et al. 2007).

Recently, a comprehensive study of SNF chemopreventive effect on three breast cancer showed that SFN provoked cell cycle arrest and senescence are mediated by epigenetic changes, namely global DNA hypomethylation, decreased levels of DNMT1 and DNMT3B, and changes in microRNA profile in all studied cancer cells. Moreover, SFN induced a decrease in m6A RNA methylation pattern that is also considered as an epigenetic regulation at the RNA level, recently discovered. So, SFN may promote genetic instability directly or indirectly by SFNmediated DNA hypomethylation and/or diminution in m6A RNA methylation pools (Lewinska et al. 2017). Interestingly, in another study, authors demonstrated that SNF upregulates miR-140, which is a negative regulator of cancer stem cell formation in basal-like early stage breast cancer. These results highlight its potential preventive properties for breast cancer (Schnekenburger and Diederich 2015). SNF represents one example of bioactive molecules from 


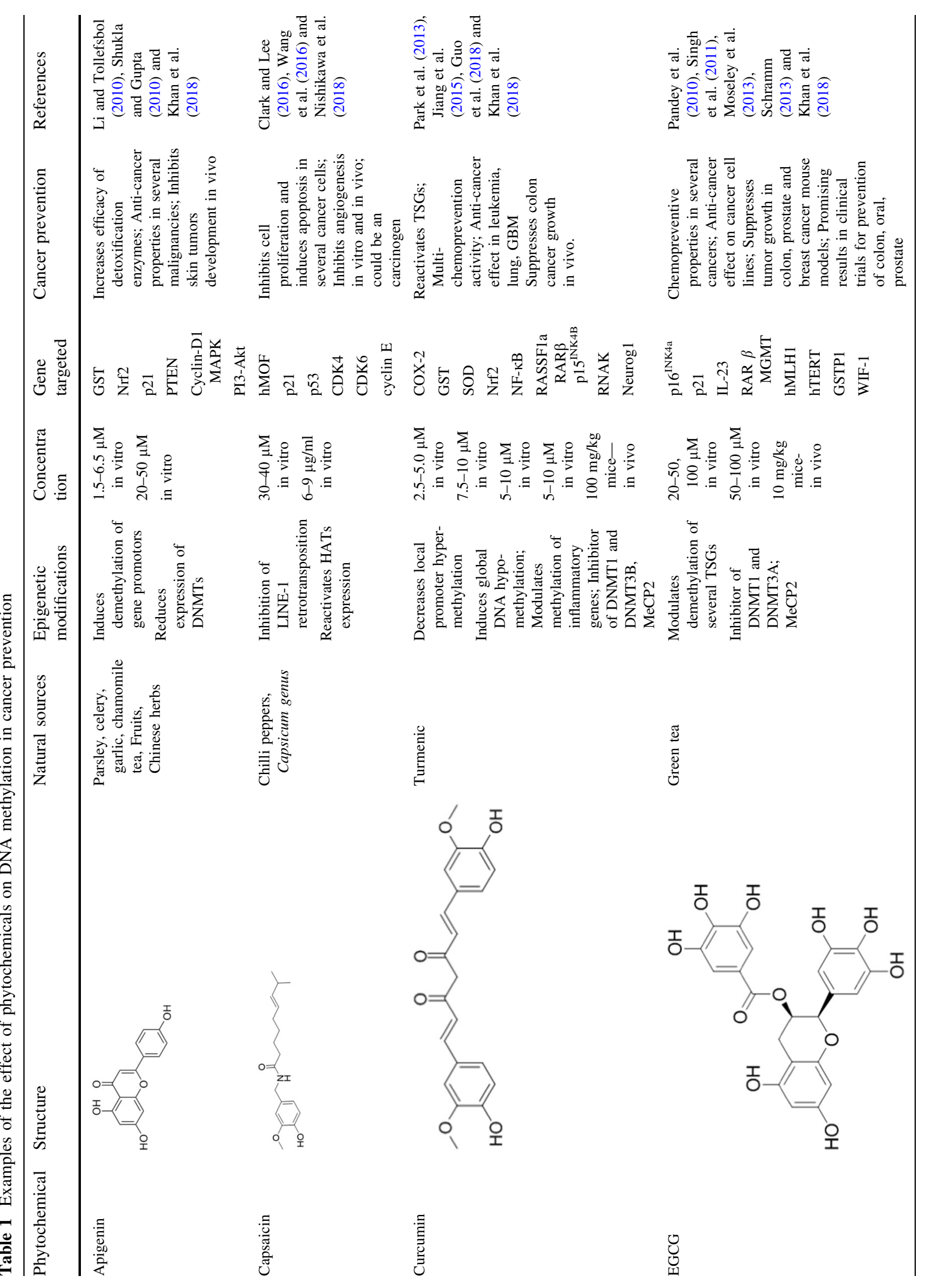




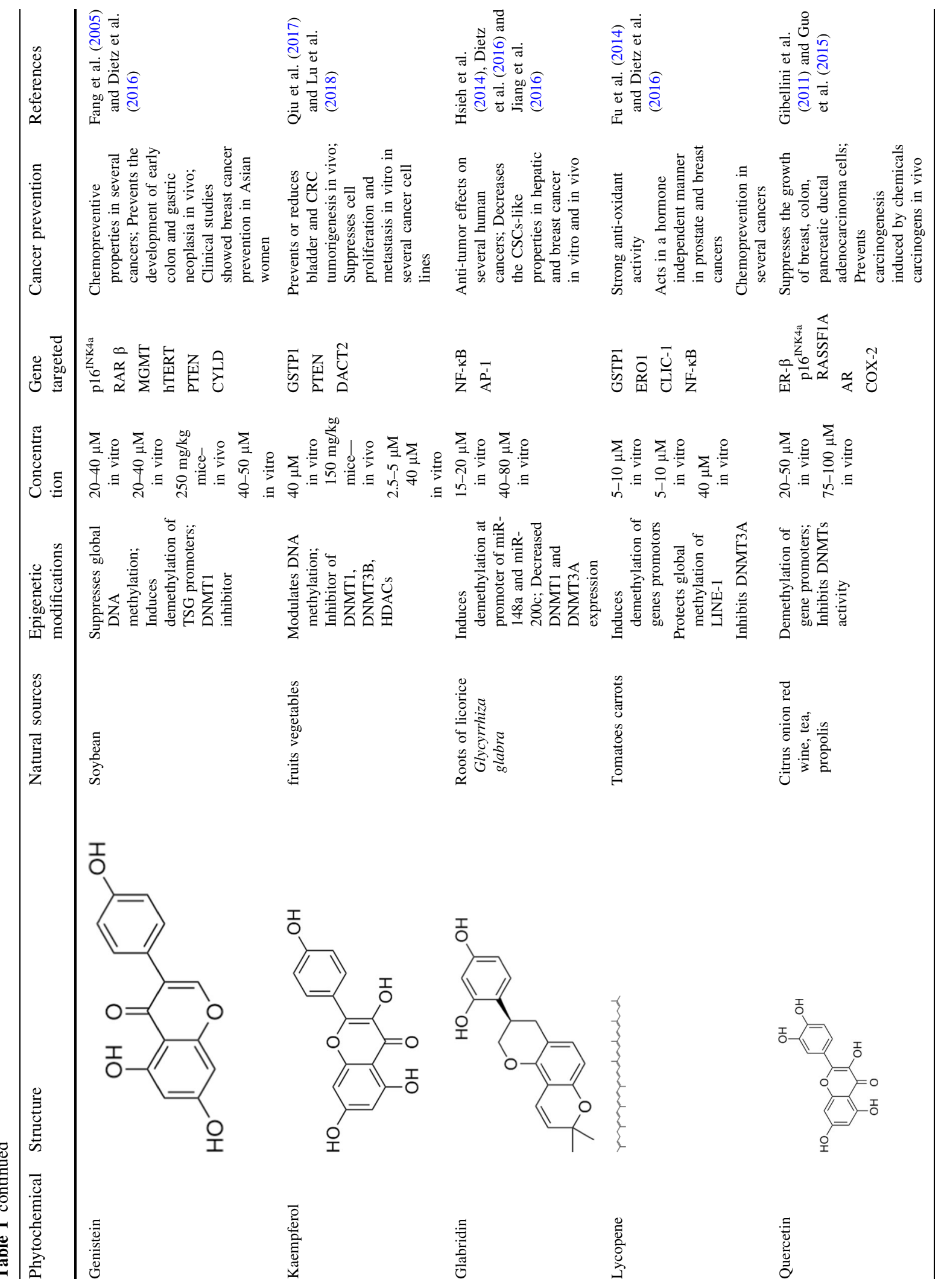




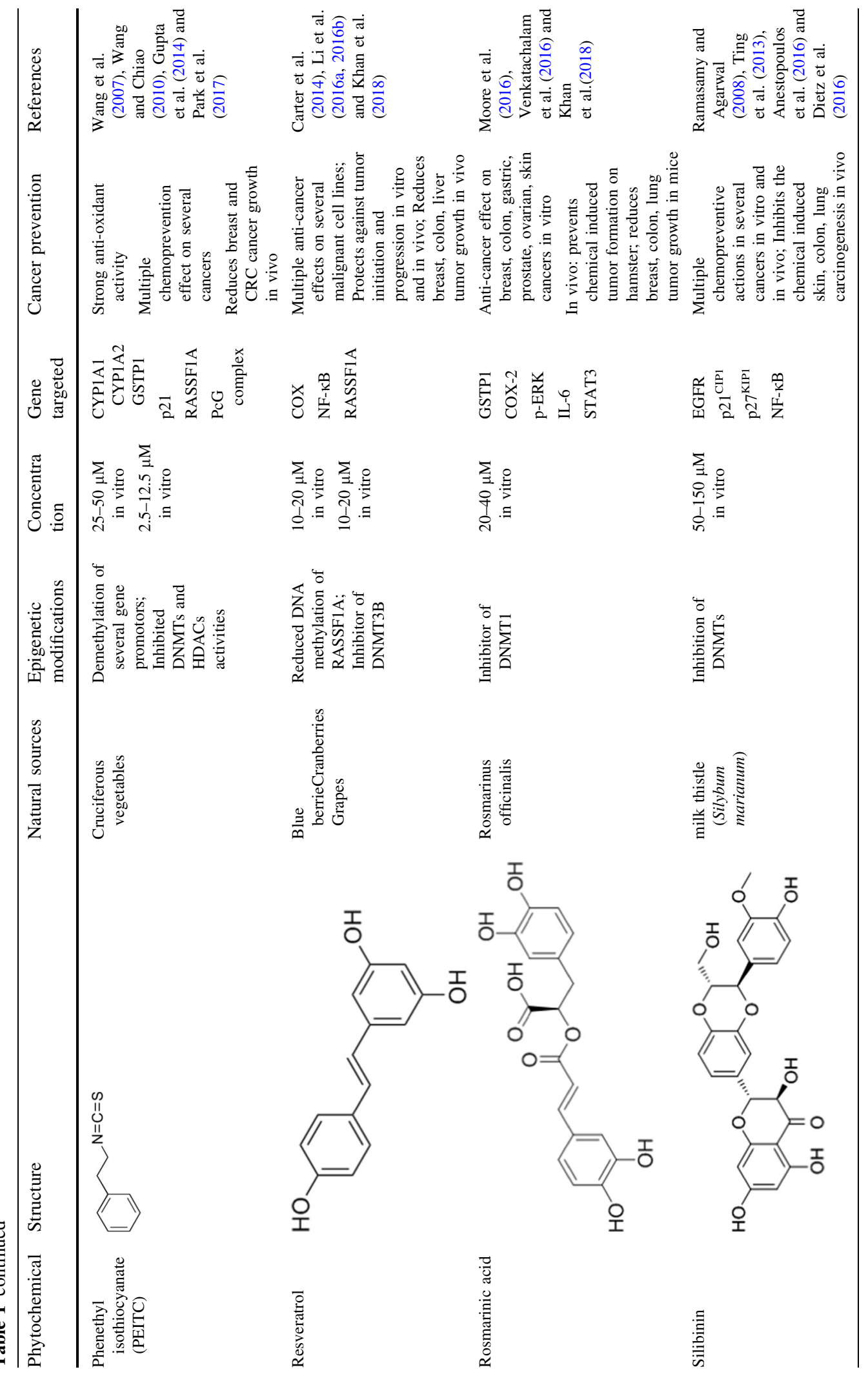




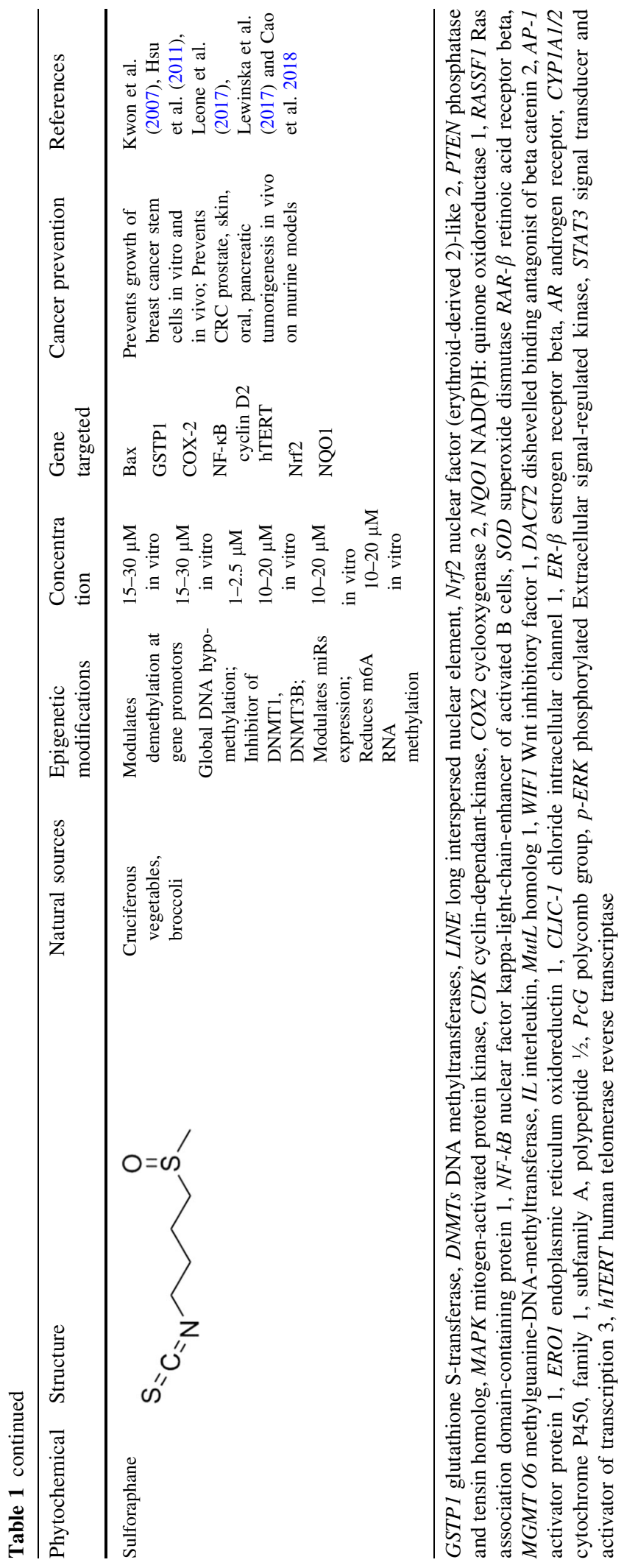




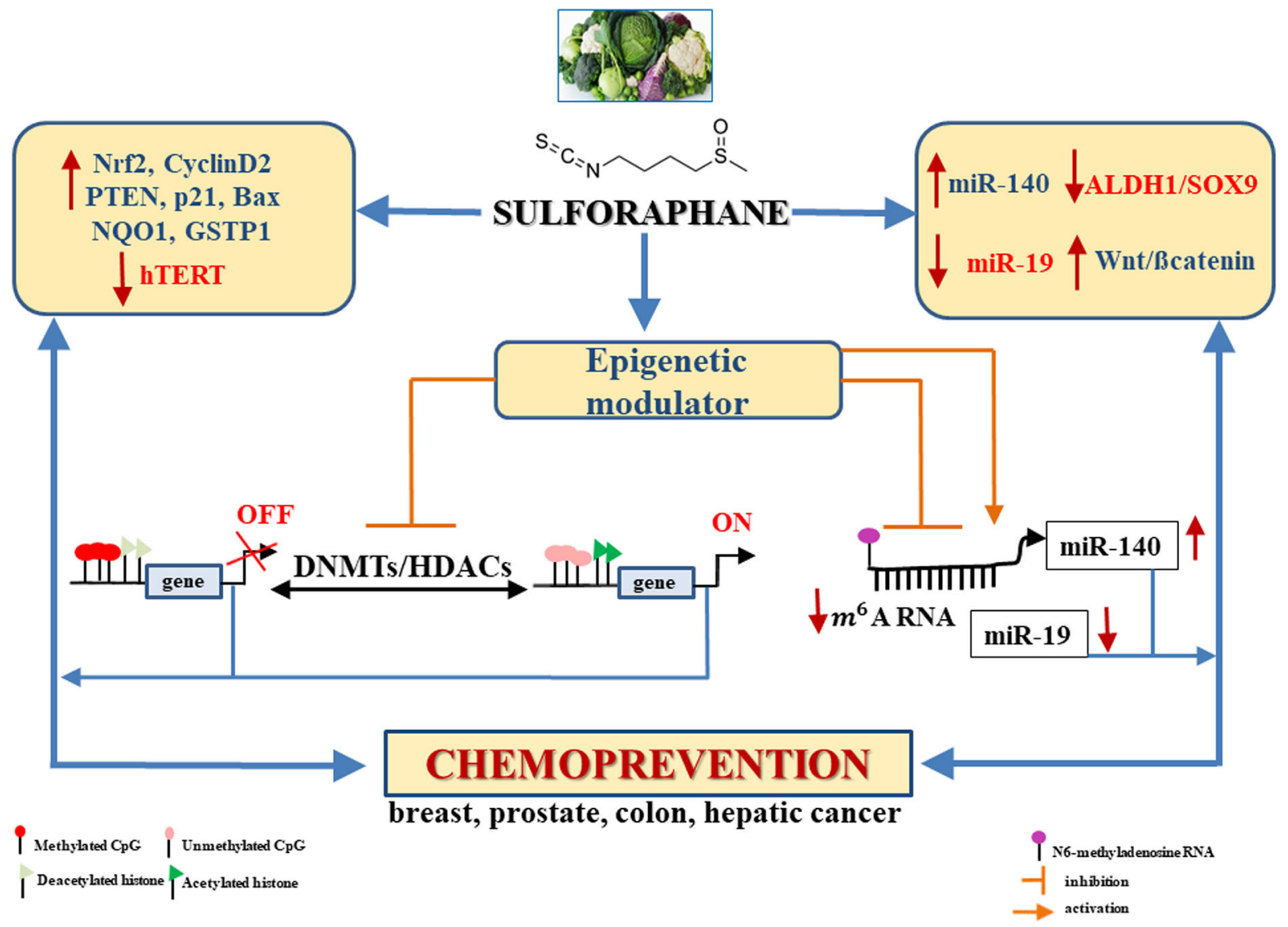

Fig. 3 Sulforaphane modulates epigenetic mechanisms in chemoprevention. Sulforaphane induces activation of Nrf2 gene and upregulates the expression of anti-oxidative enzymes, related with cancer prevention mechanism. Accumulating evidence suggests that the anti-cancer properties of sulforaphane could be at least partially mediated by its effect on

natural sources that can modulate different epigenetic mechanisms in order to restore the normal function of genes involved in chemoprevention processes, as we show in Fig. 3.

To conclude, evidence-based data from both preclinical and clinical trials are now adding to support the benefits of bioactive compounds in preventing or mitigate tumor growth. From the multiple processes altered in tumor cells, epigenetic ones, namely DNA methylation, can be modulated with the help of phytochemicals commonly found in natural foods and spices. In support of such arguments, SNF holds a strong case, showing new beneficial sides of its well documented anti-oxidant activity. Whether these active compounds will continue to be only treatment adjuvants or will seize the lead in antitumor therapy, epigenetic mechanisms. Sulforaphane is a well-described DNMTs and HDACs inhibitor, reducing gene promoter-specific methylation and increasing total and promoter-specific histone acetylation in cancer cells. Also, SFN can modulate the expression of several microRNAs, and at mRNA level it was associated with decreased 6-adenosine RNA methylation

remains to be established by large cohort and epidemiologic studies. Substantial experimental evidence indicates the potential importance of dietary and bioactive compounds in cancer prevention, but identifying direct relationships between diet and cancer in observational epidemiological studies and intervention trials had proven challenging. Study design issues, imprecise dietary assessments, and a lack of consideration of tumor heterogeneity generally attenuate relative-risk estimates in observational studies; dietary biomarkers and characterization of etiological subtypes of cancers can help to better identify diet-cancer associations. 


\section{Conclusions}

Epigenetic changes, such as DNA methylation, can be heritable, but are also influenced throughout life by environmental factors, such as diet, thus providing a novel avenue for lifestyle or therapeutic interventions. Unlike the conventional drugs, phytochemicals have multiple targets and are thus of potential value in diseases like cancer, where multiple pathways are altered. Moreover, they have selective toxicity targeting the cancer cells while showing negligible damage to normal cells. As demonstrated from taxol to sulforaphane, there is an unprecedented potential in exploring the herbal diversity for anti-cancer drug candidates. DNMT inhibitors that are currently used in clinical trials are non-selective cytosine analogues with considerable cytotoxic side effects. Several natural products, such as EGCG, curcumin, sulforaphane, from diverse chemical classes, have shown DNMT inhibitory activity, but this property needs more in-depth investigations.

Pre-clinical and clinical studies addressing the relevance and validity of in vitro experimental outcomes as well as analysis of safety profile, dose, and length of treatment are to be validated by future trials. New technologies and advances in genetics, epigenetics and metabolomics, and consideration of the influence of the microbiome, will expand our understanding of the role of dietary phytochemicals in cancer risk and disease progression.

Whilst it may be impossible to avoid the initiating mutagenesis, cancer could be prevented if cells bearing the initiating lesion(s) could be identified and the faulty epigenetic process corrected with the help of active phytochemicals. The epigenetic modulation of the early cancer events discovered in humans using phytochemicals remains a hope for cancer therapy.

Acknowledgements This work was supported by the grant COP A 1.2.3., ID: P_40_197/2016 and by Ministry of Research and Innovation in Romania, under Program 1-The Improvement of the National System of Research and Development, Subprogram 1.2-Institutional ExcellenceProjects of Excellence Funding in RDI: Contract PERFORM No. 22PFE/2018 and Contract No. 7PFE/16.10.2018. The authors would like to thank Ms. Irina Radu, certified translator in Medicine and Pharmacy (certificate credentials: series E no. 0048), for professional linguistic assistance.

\section{Compliance with ethical standards}

Conflict of interest The authors confirm that there are no conflicts of interest.

Open Access This article is distributed under the terms of the Creative Commons Attribution 4.0 International License (http:// creativecommons.org/licenses/by/4.0/), which permits unrestricted use, distribution, and reproduction in any medium, provided you give appropriate credit to the original author(s) and the source, provide a link to the Creative Commons license, and indicate if changes were made.

\section{References}

Anand P, Kunnumakkara AB, Kunnumakara AB et al (2008) Cancer is a preventable disease that requires major lifestyle changes. Pharm Res 25:2097-2116

Anestopoulos I, Sfakianos AP, Franco R et al (2016) A novel role of silibinin as a putative epigenetic modulator in human prostate carcinoma. Molecules 22:62

Anwar SL, Lehmann U (2014) DNA methylation, microRNAs, and their crosstalk as potential biomarkers in hepatocellular carcinoma. World J Gastroenterol 20:7894-7913

Banerji N (2017) Organic photovoltaics: pushing the knowledge of interfaces. Nat Mater 16(5):503-505. https://doi.org/10. 1038/nmat4871

Bannister AJ, Kouzarides T (2011) Regulation of chromatin by histone modifications. Cell Res 21:381-395

Baylin SB, Herman JG (2000) DNA hypermethylation in tumorigenesis: epigenetics joins genetics. Trends Genet 16:168-174

Bintu L, Yong J, Antebi YE et al (2016) Dynamics of epigenetic regulation at the single-cell level. Science 351:720-724

Bistulfi G, Vandette E, Matsui S, Smiraglia DJ (2010) Mild folate deficiency induces genetic and epigenetic instability and phenotype changes in prostate cancer cells. BMC Biol 8:6. https://doi.org/10.1186/1741-7007-8-6

Busch C, Burkard M, Leischner C, Lauer UM, Frank J, Venturelli $S$ (2015) Epigenetic activities of flavonoids in the prevention and treatment of cancer. Clin Epigenetics 7:64

Butcher DT, Rodenhiser DI (2007) Epigenetic inactivation of BRCA1 is associated with aberrant expression of CTCF and DNA methyltransferase (DNMT3B) in some sporadic breast tumours. Eur J Cancer 43:210-219

Cao S, Wang L, Zhang Z et al (2018) Sulforaphane-induced metabolomic responses with epigenetic changes in estrogen receptor positive breast cancer cells. FEBS Open Bio 8:2022-2034

Carter LG, D'Orazio JA, Pearson KJ (2014) Resveratrol and cancer: focus on in vivo evidence. Endocr Relat Cancer 21:R209-R225

Chen Z, Riggs AD (2011) DNA methylation and demethylation in mammals. J Biol Chem 286:18347-18353

Chen NC, Yang F, Capecci LM, Gu Z, Schafer AI, Durante W, Yang XF, Wang H (2010) Regulation of homocysteine metabolism and methylation in human and mouse tissues. FASEB 24:2804-2817 
Clark R, Lee S-H (2016) Anticancer properties of capsaicin against human cancer. Anticancer Res 36:837-843

Dawson MA, Kouzarides T (2012) Cancer epigenetics: from mechanism to therapy. Cell 150:12-27

De Carvalho DD, Sharma S, You JS et al (2012) DNA methylation screening identifies driver epigenetic events of cancer cell survival. Cancer Cell 21:655-667

Deaton AM, Bird A (2011) CpG islands and the regulation of transcription. Genes Dev 25:1010-1022

Dietz BM, Hajirahimkhan A, Dunlap TL et al (2016) Botanicals and their bioactive phytochemicals for women's health. Pharmacol Rev 68:1026-1073

Donohoe DR, Bultman SJ (2012) Metaboloepigenetics: interrelationships between energy metabolism and epigenetic control of gene expression. J Cell Physiol 227:3169-3177

Du GJ, Zhang Z, Wen XD, Yu C, Calway T, Yuan CS, Wang CZ (2012) Epigallocatechin Gallate (EGCG) is the most effective cancer chemopreventive polyphenol in green tea. Nutrients 4:1679-1691

Edmands WM, Ferrari P, Rothwell JA et al (2015) Polyphenol metabolome in human urine and its association with intake of polyphenol-rich foods across European countries. Am J Clin Nutr 102:905-913

Fang MZ, Wang Y, Ai N, et al (2003) Tea polyphenol (-)epigallocatechin-3-gallate inhibits DNA methyltransferase and reactivates methylation-silenced genes in cancer cell lines. Cancer Res 63(22):7563-7570

Fang MZ, Chen D, Sun Y, Jin Z, Christman JK, Yang CS (2005) Reversal of hypermethylation and reactivation of p16INK4a, RARbeta, and MGMT genes by genistein and other isoflavones from soy. Clin Cancer Res 11:7033-7041

Feinberg AP, Ohlsson R, Henikoff S (2006) The epigenetic progenitor origin of human cancer. Nat Rev Genet 7:21-33

Feinberg AP, Koldobskiy MA, Göndör A (2016) Epigenetic modulators, modifiers and mediators in cancer aetiology and progression. Nat Rev Genet 17:284-299

Ficz G, Branco MR, Seisenberger S et al (2011) Dynamic regulation of 5-hydroxymethylcytosine in mouse ES cells and during differentiation. Nature 473:398-402

Fu L-J, Ding Y-B, Wu L-X et al (2014) The effects of lycopene on the methylation of the GSTP1 promoter and global methylation in prostatic cancer cell lines PC3 and LNCaP. Int J Endocrinol 2014:620165

Gibellini L, Pinti M, Nasi M et al (2011) Quercetin and cancer chemoprevention. Evid Based Complement Alternat Med 2011:591356

Guo Y, Su Z-Y, Kong A-NT (2015) Current perspectives on epigenetic modifications by dietary chemopreventive and herbal phytochemicals. Curr Pharmacol Rep 1:245-257

Guo Y, Wu R, Gaspar JM et al (2018) DNA methylome and transcriptome alterations and cancer prevention by curcumin in colitis-accelerated colon cancer in mice. Carcinogenesis 39:669-680

Gupta P, Wright SE, Kim S-H et al (2014) Phenethyl isothiocyanate: a comprehensive review of anti-cancer mechanisms. Biochim Biophys Acta 1846:405-424

Hardman WE (2014) Diet components can suppress inflammation and reduce cancer risk. Nutr Res Pract $8(3): 233-240$
Henning SM, Wang P, Carpenter CL, Heber D (2013) Epigenetic effects of green tea polyphenols in cancer. Epigenomics 5(6):729-741

Ho E, Beaver LM, Williams DE, Dashwood RH (2011) Dietary factors and epigenetic regulation for prostate cancer prevention. Adv Nutr 2(6):497-510

Hsieh M-J, Lin C-W, Yang S-F et al (2014) Glabridin inhibits migration and invasion by transcriptional inhibition of matrix metalloproteinase 9 through modulation of NF- $\kappa \mathrm{B}$ and AP-1 activity in human liver cancer cells. Br J Pharmacol 171:3037-3050

Hsu A, Wong CP, Yu Z et al (2011) Promoter de-methylation of cyclin D2 by sulforaphane in prostate cancer cells. Clin Epigenetics 3:3

Jerónimo C, Bastian PJ, Bjartell A et al (2011) Epigenetics in prostate cancer: biologic and clinical relevance. Eur Urol 60:753-766

Jiang A, Wang X, Shan X et al (2015) Curcumin reactivates silenced tumor suppressor gene RAR $\beta$ by reducing DNA methylation. Phytother Res 29:1237-1245

Jiang F, Li Y, Mu J et al (2016) Glabridin inhibits cancer stem cell-like properties of human breast cancer cells: an epigenetic regulation of miR-148a/SMAd2 signaling. Mol Carcinog 55:929-940

Jones PA (2012) Functions of DNA methylation: Islands, start sites, gene bodies and beyond. Nat Rev Genet 13:484-492

Jones PA, Baylin SB (2007) The epigenomics of cancer. Cell 128:683-692

Keshet I, Schlesinger Y, Farkash S et al (2006) Evidence for an instructive mechanism of de novo methylation in cancer cells. Nat Genet 38:149-153

Khan MI, Rath S, Adhami VM et al (2018) Targeting epigenome with dietary nutrients in cancer: current advances and future challenges. Pharmacol Res 129:375-387

Kim JH, Dhanasekaran SM, Prensner JR et al (2011) Deep sequencing reveals distinct patterns of DNA methylation in prostate cancer. Genome Res 21:1028-1041

Kim SJ, Zhao H, Hardikar S et al (2013) A DNMT3A mutation common in AML exhibits dominant-negative effects in murine ES cells. Blood 122:4086-4089

Kitkumthorn N, Mutirangura A (2011) Long interspersed nuclear element-1 hypomethylation in cancer: biology and clinical applications. Clin Epigenetics 2:315-330

Kottakis F, Nicolay BN, Roumane A et al (2016) LKB1 loss links serine metabolism to DNA methylation and tumorigenesis. Nature 539:390-395

Kozaki K, Inazawa J (2012) Tumor-suppressive microRNA silenced by tumor-specific DNA hypermethylation in cancer cells. Cancer Sci 103:837-845

Kumar S, Pandey AK (2013) Chemistry and biological activities of flavonoids: an overview. ScientificWorldJournal 2013:162750. https://doi.org/10.1155/2013/162750

Kwon KH, Barve A, Yu S et al (2007) Cancer chemoprevention by phytochemicals: potential molecular targets, biomarkers and animal models. Acta Pharmacol Sin 28:1409-1421

Lee WJ, Shim JY, Zhu BT (2005) Mechanisms for the inhibition of DNA methyltransferases by tea catechins and bioflavonoids. Mol Pharmacol 68(4):1018-1030

Lee E, Iskow R, Yang L et al (2012) Landscape of somatic retrotransposition in human cancers. Science 337:967-971 
Leone A, Diorio G, Sexton W et al (2017) Sulforaphane for the chemoprevention of bladder cancer: molecular mechanism targeted approach. Oncotarget 8:35412-35424

Lewinska A, Adamczyk-Grochala J, Deregowska A et al (2017) Sulforaphane-induced cell cycle arrest and senescence are accompanied by DNA hypomethylation and changes in microRNA profile in breast cancer cells. Theranostics 7:3461-3477

Li Y, Tollefsbol TO (2010) Impact on DNA methylation in cancer prevention and therapy by bioactive dietary components. Curr Med Chem 17(20):2141-2151

Li Y, Liu L, Andrews LG et al (2009) Genistein depletes telomerase activity through cross-talk between genetic and epigenetic mechanisms. Int J Cancer 125(2):286-296

Li W, Pung D, Su ZY et al (2016a) Epigenetics reactivation of Nrf2 in prostate TRAMP C1 cells by curcumin analogue FN1. Chem Res Toxicol 29(4):694-703

Li W, Guo Y, Zhang C et al (2016b) Dietary phytochemicals and cancer chemoprevention: a perspective on oxidative stress, inflammation, and epigenetics. Chem Res Toxicol 29:2071-2095

Lian CG, Xu Y, Ceol C et al (2012) Loss of 5-hydroxymethylcytosine is an epigenetic hallmark of melanoma. Cell 150:1135-1146

Liang G, Chan MF, Tomigahara Y et al (2002) Cooperativity between DNA methyltransferases in the maintenance methylation of repetitive elements. Mol Cell Biol 22:480-491

Lu L, Wang Y, Ou R et al (2018) DACT2 epigenetic stimulator exerts dual efficacy for colorectal cancer prevention and treatment. Pharmacol Res 129:318-328

Maddocks OD, Labuschagne CF, Adams PD et al (2016) Serine metabolism supports the methionine cycle and DNA/RNA methylation through de novo ATP synthesis in cancer cells. Mol Cell 61(2):210-221

Mellén M, Ayata P, Dewell S et al (2012) MeCP2 binds to 5hmC enriched within active genes and accessible chromatin in the nervous system. Cell 151:1417-1430

Memedula S, Belmont AS (2003) Sequential recruitment of HAT and SWI/SNF components to condensed chromatin by VP16. Curr Biol 13:241-246

Moore J, Yousef M, Tsiani E (2016) Anticancer effects of rosemary (Rosmarinus officinalis L.) extract and rosemary extract polyphenols. Nutrients 8(11):731-763

Mortusewicz O, Schermelleh L, Walter J et al (2005) Recruitment of DNA methyltransferase I to DNA repair sites. Proc Natl Acad Sci USA 102:8905-8909

Moseley VR, Morris J, Knackstedt RW et al (2013) Green tea polyphenol epigallocatechin 3-gallate, contributes to the degradation of DNMT3A and HDAC3 in HCT 116 human colon cancer cells. Anticancer Res 33:5325-5333

Myzak MC, Tong P, Dashwood W-M et al (2007) Sulforaphane retards the growth of human PC-3 xenografts and inhibits HDAC activity in human subjects. Exp Biol Med (Maywood) 232:227-234

Naponelli V, Ramazzina I, Lenzi C et al (2017) Green tea catechins for prostate cancer prevention: present achievements and future challenges. Antioxidants (Basel) 6(2):1-23. https://doi.org/10.3390/antiox6020026

Newman DJ, Cragg GM (2016) Natural products as sources of new drugs from 1981 to 2014. J Nat Prod 79:629-661
Newman AC, Maddocks ODK (2017) One-carbon metabolism in cancer. Br J Cancer 116:1499-1504

Niculescu MD, Zeisel SH (2002) Diet, methyl donors and DNA methylation: interactions between dietary folate, methionine and choline. J Nutr 132(8 Suppl):2333S-2335S

Nishikawa Y, Nakayama R, Obika S et al (2018) Inhibition of LINE-1 retrotransposition by capsaicin. Int $\mathrm{J}$ Mol Sci 19:3243. https://doi.org/10.3390/ijms19103243

Oswald J, Engemann S, Lane N et al (2000) Active demethylation of the paternal genome in the mouse zygote. Curr Biol 10:475-478

Pandey M, Shukla S, Gupta S (2010) Promoter demethylation and chromatin remodeling by green tea polyphenols leads to re-expression of GSTP1 in human prostate cancer cells. Int J Cancer 126:2520-2533

Parasramka MA, Ho E, Williams DE et al (2012) MicroRNAs, diet, and cancer: new mechanistic insights on the epigenetic actions of phytochemicals. Mol Carcinog 51:213-230

Park W, Amin ARMR, Chen ZG et al (2013) New perspectives of curcumin in cancer prevention. Cancer Prev Res (Phila) $6: 387-400$

Park JE, Sun Y, Lim SK et al (2017) Dietary phytochemical PEITC restricts tumor development via modulation of epigenetic writers and erasers. Sci Rep 7:40569

Plass C, Pfister SM, Lindroth AM et al (2013) Mutations in regulators of the epigenome and their connections to global chromatin patterns in cancer. Nat Rev Genet 14:765-780

Pogribny IP, Ross SA, Wise C et al (2006) Irreversible global DNA hypomethylation as a key step in hepatocarcinogenesis induced by dietary methyl deficiency. Mutat Res 593(1-2):80-87

Pop S, Enciu A-M, Necula LG et al (2018) Long non-coding RNAs in brain tumours: focus on recent epigenetic findings in glioma. J Cell Mol Med 22:4597-4610

Qiu W, Lin J, Zhu Y et al (2017) Kaempferol modulates DNA methylation and downregulates DNMT3B in bladder cancer. Cell Physiol Biochem 41:1325-1335

Ramasamy K, Agarwal R (2008) Multitargeted therapy of cancer by silymarin. Cancer Lett 269:352-362

Remely M, Lovrecic L, de la Garza AL et al (2015) Therapeutic perspectives of epigenetically active nutrients. Br J Pharmacol 172:2756-2768

Rollins RA, Haghighi F, Edwards JR et al (2006) Large-scale structure of genomic methylation patterns. Genome Res 16:157-163

Schnekenburger M, Diederich M (2015) Nutritional epigenetic regulators in the field of cancer. In: Epigenetic cancer therapy. Elsevier, pp 393-425

Schramm L (2013) Going green: the role of the green tea component EGCG in chemoprevention. J Carcinog Mutagen 4:1000142

Sharma S, Kelly TK, Jones PA (2010) Epigenetics in cancer. Carcinogenesis 31:27-36

Shen H, Laird PW (2013) Interplay between the cancer genome and epigenome. Cell 153:38-55

Shukla S, Gupta S (2010) Apigenin: a promising molecule for cancer prevention. Pharm Res 27:962-978

Singh BN, Shankar S, Srivastava RK (2011) Green tea catechin, epigallocatechin-3-gallate (EGCG): mechanisms, perspectives and clinical applications. Biochem Pharmacol 82:1807-1821 
Slotkin RK, Martienssen R (2007) Transposable elements and the epigenetic regulation of the genome. Nat Rev Genet $8: 272-285$

Sporn MB (2011) Perspective: the big C-for Chemoprevention. Nature 471:S10-S11

Starczynowski DT, Morin R, McPherson A et al (2011) Genome-wide identification of human microRNAs located in leukemia-associated genomic alterations. Blood 117:595-607

Stefanska B, Karlic H, Varga F et al (2012) Epigenetic mechanisms in anti-cancer actions of bioactive food components-the implications in cancer prevention. $\mathrm{Br} \mathrm{J}$ Pharmacol 167:279-297

Sun L, Subar AF, Bosire C et al (2017) Dietary flavonoid intake reduces the risk of head and neck but not esophageal or gastric cancer in US men and women. J Nutr 147:1729-1738

Suzuki MM, Bird A (2008) DNA methylation landscapes: provocative insights from epigenomics. Nat Rev Genet 9:465-476

Taby R, Issa J-P (2010) Cancer epigenetics. CA Cancer J Clin 60:376-392

Timp W, Feinberg AP (2013) Cancer as a dysregulated epigenome allowing cellular growth advantage at the expense of the host. Nat Rev Cancer 13:497-510

Ting H, Deep G, Agarwal R (2013) Molecular mechanisms of silibinin-mediated cancer chemoprevention with major emphasis on prostate cancer. AAPS J 15:707-716

Tucker DW, Getchell CR, McCarthy ET et al (2018) Epigenetic reprogramming strategies to reverse global loss of 5-hydroxymethylcytosine, a prognostic factor for poor survival in high-grade serous ovarian cancer. Clin Cancer Res 24:1389-1401

Venkatachalam K, Gunasekaran S, Namasivayam N (2016) Biochemical and molecular mechanisms underlying the chemopreventive efficacy of rosmarinic acid in a rat colon cancer. Eur J Pharmacol 791:37-50

Viré E, Brenner C, Deplus R et al (2006) The polycomb group protein EZH2 directly controls DNA methylation. Nature 439:871-874

Wang LG, Chiao JW (2010) Prostate cancer chemopreventive activity of phenethyl isothiocyanate through epigenetic regulation (review). Int J Oncol 37:533-539
Wang LG, Beklemisheva A, Liu XM et al (2007) Dual action on promoter demethylation and chromatin by an isothiocyanate restored GSTP1 silenced in prostate cancer. Mol Carcinog 46:24-31

Wang F, Zhao J, Liu D et al (2016) Capsaicin reactivates hMOF in gastric cancer cells and induces cell growth inhibition. Cancer Biol Ther 17:1117-1125

Weaver ICG, Cervoni N, Champagne FA et al (2004) Epigenetic programming by maternal behavior. Nat Neurosci $7: 847-854$

Weisenberger DJ (2014) Characterizing DNA methylation alterations from The Cancer Genome Atlas. J Clin Invest 124:17-23

Williams K, Christensen J, Pedersen MT et al (2011) TET1 and hydroxymethylcytosine in transcription and DNA methylation fidelity. Nature 473:343-348

Witte T, Plass C, Gerhauser C (2014) Pan-cancer patterns of DNA methylation. Genome Med 6:66

Wu X, Zhang Y (2017) TET-mediated active DNA demethylation: mechanism, function and beyond. Nat Rev Genet 18:517-534

You JS, Jones PA (2012) Cancer genetics and epigenetics: Two sides of the same coin? Cancer Cell 22:9-20

Yu D-H, Waterland RA, Zhang P et al (2014) Targeted p16(Ink4a) epimutation causes tumorigenesis and reduces survival in mice. J Clin Invest 124:3708-3712

Zamora-Ros R, Touillaud M, Rothwell JA et al (2014) Measuring exposure to the polyphenol metabolome in observational epidemiologic studies: current tools and applications and their limits. Am J Clin Nutr 100:11-26

Zhao Z, Wu Q, Cheng J et al (2010) Depletion of DNMT3A suppressed cell proliferation and restored PTEN in hepatocellular carcinoma cell. J Biomed Biotechnol 2010:737535

Zhu BT, Wu KY, Wang P et al (2010) O-methylation of catechol estrogens by human placental catechol- $O$-methyltransferase: interindividual differences in sensitivity to heat inactivation and to inhibition by dietary polyphenols. Drug Metab Dispos 38(10):1892-1899

Publisher's Note Springer Nature remains neutral with regard to jurisdictional claims in published maps and institutional affiliations. 